\title{
RR Lyrae and short-period variable stars in the dwarf irregular galaxy NGC 6822 ${ }^{1}$
}

\author{
Gisella Clementini, ${ }^{2}$ Enrico V. Held, ${ }^{3}$ Lara Baldacci, ${ }^{2,4}$ Luca Rizzi ${ }^{3,5}$
}

\begin{abstract}
We report the discovery of a large number of short-period variable stars in the dwarf irregular galaxy NGC 6822, based on deep time-series imaging carried out with the ESO Very Large Telescope. In particular, we found a modest population of RR Lyrae stars tracing the presence of an old stellar component in NGC 6822. Measurements of the average luminosity of RR Lyrae stars provide a new independent estimate of the distance to this galaxy based on a Pop. II indicator, $(m-M)_{0}=23.36 \pm 0.17$. In addition, our new data show a significant population of small-amplitude, short-period variable stars filling the instability strip starting at luminosities only a few tenths of a magnitude brighter than the RR Lyrae stars. Given the presence of an extended star formation in NGC 6822, the faint end of this distribution of short-period variable stars is likely to originate from a population of intermediate-age, metal-poor He-burning stars, younger and more massive than RR Lyrae stars.
\end{abstract}

Subject headings: galaxies: dwarf — galaxies: individual (NGC 6822) — galaxies: irregular — Local Group — stars: variable: other

\section{Introduction}

Extended horizontal branches (HBs) and/or significant populations of RR Lyrae stars have been detected in several dwarf galaxies (NGC 147: Saha \& Hoessel 1987, Saha et al.

\footnotetext{
${ }^{1}$ Based on data collected at E.S.O. La Silla, Chile, Proposal No. 67B-0557

${ }^{2}$ INAF, Osservatorio Astronomico di Bologna, via Ranzani 1, I-40127 Bologna, Italy; (gisella, baldacci)@bo.astro.it

${ }^{3}$ INAF, Osservatorio Astronomico di Padova, vicolo dell'Osservatorio 5, I-35122 Padova, Italy; (held, rizzi)@pd.astro.it

${ }^{4}$ Dipartimento di Astronomia, Università di Bologna, via Ranzani 1, I-40127 Bologna, Italy

${ }^{5}$ Dipartimento di Astronomia, Università di Padova, vicolo dell'Osservatorio 2, I-35122 Padova, Italy
} 
1990; NGC 185: Saha \& Hoessel 1990; NGC 205: Saha et al. 1992b; WLM: Rejkuba et al. 2000; Leo I: Held et al. 2000, 2001; IC 1613: Saha et al. 1992a, Dolphin et al. 2001; Leo A: Dolphin et al. 2002, Schulte-Ladbeck et al. 2002; And VI: Pritzl et al. 2002). These observations provide evidence for a first epoch of star formation which is common to the majority of Local Group dwarf galaxies, both star forming and presently quiescent.

The presence of an old stellar population is still an open question in NGC6822, the nearest dwarf irregular (dI) galaxy (and the closest star-forming galaxy beyond the Magellanic Clouds). Based on its luminosity, gas fraction, metallicity, and star formation rate, NGC 6822 appears to be a typical, relatively gently star-forming dI galaxy. Several studies addressed its star formation history by modeling its color-magnitude diagram ( CMD) (see, e.g., Marconi et al. 1995; Gallart et al. 1996b; Wyder 2001; and references therein). These studies suggested that NGC 6822 most likely began forming stars 12-15 Gyr ago from lowmetallicity gas. However, the data are not inconsistent with star formation starting 6-9 Gyr ago from a relatively metal enriched interstellar medium.

The discovery of RR Lyrae variable stars in NGC 6822 presented in this Letter, breaks the uncertainty between these two scenarios by demonstrating the presence of an old (> 11 Gyr), metal-poor stellar population. The average magnitude of the newly discovered RR Lyrae variable stars is also used to derive a new estimate of the distance to this galaxy based on a Pop. II indicator.

\section{Observations and data reduction}

\subsection{Observations}

Time-series imaging of NGC 6822 was obtained on 3 half nights on Aug. 15, 16, and 20, 2001 using the focal reducer FORS2 on the ESO VLT/UT3 telescope at Cerro Paranal, Chile. The standard resolution collimator was used, yielding a $6.8 \times 6.8$ arcmin field-of-view with a $2048^{2}$ pixel Textronix CCD (ESO \#160), read in 4-port mode with no binning.

The scheduling was devised so as to provide good coverage of the light curves for variable stars with periods between 0.2 and 0.7 days, with optimal efficiency for periods around 0.53 and 0.59-0.60 d. The observations consisted of $36 \times 900 \mathrm{~s} V$ exposures of a galaxy field located at $19^{h} 45^{m} 13.3^{s},-14^{\circ} 45^{\prime} 55^{\prime \prime}$ (offset from the center of NGC 6822 to avoid regions of active star formation). These data were complemented by $11 B$ and $1 I$ exposures of the same duration. The nights were all photometric and the seeing varied from 0.5 to 1.0 arcsec, with a median value around 0.7 arcsec. 


\subsection{Photometry}

The FORS2 images were reduced in a standard way using the XCCDRED IRAF ${ }^{6}$ package. Photometry of all bias-subtracted, flat-fielded images was obtained using ALLFRAME (Stetson 1994). Since our standard stars were mostly saturated even with the shortest exposure times allowed by the instrument software, the instrumental $b, v$, and $i$ magnitudes were calibrated using the color equations available from the FORS Web page. The photometric zero points were then set by comparison with secondary standard stars established in the field using Wide Field Imager observations at the $2.2 \mathrm{~m}$ ESO/MPI telescope, accurately calibrated onto the Landolt (1992) system (L. Rizzi et al. 2003, in prep.). The estimated uncertainty of the zero point calibration is $0.04 \mathrm{mag}$ in both $B$ and $V$.

\subsection{Variable star identification and period search}

Candidate variable stars were identified using ISIS2.1 (Alard 2000), a package specifically designed for the detection of variable stars in crowded fields. ISIS is based on an optimal image subtraction technique. The variable star detection procedure consists of the following steps: (i) geometric alignment of the image series and re-mapping of each image to the same grid; (ii) construction of a reference image obtained by stacking a subset of images; (iii) subtraction of each individual frame from the reference image convolved with a suitable kernel to match seeing variations and geometrical distortions on the individual images; (iv) photometry of the variable objects on the difference images, in terms of differential fluxes. The search performed by ISIS on this image yielded a master list of 448 candidate variable stars. Candidate variable stars were then counter-identified on the ALLFRAME $B, V$ master catalogue, where about $2 / 3$ of the objects flagged by ISIS as variable sources were found.

All stars in the candidate master list were then analyzed to confirm their variable star nature and derive light curves. For the analysis, we combined the time series obtained from ALLFRAME photometry (whenever available) and from ISIS differential flux measurements. In this Letter, given our primary interest in RR Lyrae variable stars, only stars with mean magnitude fainter than $V=23$ are discussed. A full census of the variable stars identified in NGC 6822 will be given in a forthcoming paper (E. V. Held et al. 2003, in prep.).

For the period search we used the program GrATiS (Graphical Analyzer of Time Series:

\footnotetext{
${ }^{6}$ IRAF is distributed by the National Optical Astronomical Observatories, which are operated by the Association of Universities for Research in Astronomy, Inc., under cooperative agreement with the National Science Foundation
} 
see Clementini et al. 2000 for details), which was run on the ISIS differential flux data converted to magnitude scale. Whenever multiple periods appeared equally compatible with the data, the star was omitted from the present "best sample". The light curves were finally calibrated to the standard photometric system by modeling the color variation along the cycle. The sampling of the $B$ data points, although sparser than that of the $V$ observations, is sufficient to constrain the model color curves quite well.

\section{Results}

\section{1. $\quad$ Light curves}

Among the 84 candidates in the faint sample considered in this Letter, 43 have well sampled light curves in both $V$ and $B$, and a scatter about the light curve model not larger than 0.1 mag. This restricted sample was found to includes 17 RR Lyrae, 20 brighter shortperiod variable stars with very similar period distributions, and 6 further variables among which some binary systems and some possible $\beta$ Cephei stars.

For the classification of the faint sample of variable stars we took into account the mean luminosity and color of the stars, and the period, amplitude, and shape of the light curve. Based on these characteristics our sample was found to include RR Lyrae stars and brighter stars pulsating both in the fundamental mode (the vast majority) and in the first overtone. The mean period of the 15 ab-type RR Lyrae variable stars with the best defined light curves is $\left\langle P_{a b}\right\rangle=0.613 \pm 0.028$, where the uncertainty is the stardard error on the mean.

Figure 1 shows some examples of the $V$ and $B$ light curves of variable stars discovered in NGC 6822. Labels in each panel give the identifier, period, amplitude and intensityaveraged magnitude $(V$ and $B$ ) of each variable star. The left panels show typical light curves of RR Lyrae variable stars, both fundamental mode (top and middle) and first overtone pulsators (c-type RR Lyrae, bottom panel). The light curves on the right panels refer to some of the numerous pulsating stars that have been discovered in NGC 6822. These variables are brighter than the RR Lyrae stars and have on average smaller amplitudes at a given period. Because of their characteristics these stars appear related to both the short-period Population I Cepheids found in dwarf irregular galaxies (Dolphin et al. 2001, 2002, 2003; Sharpee et al. 2002) and the anomalous Cepheids population in dwarf spheroidal galaxies (e.g., Nemec, Nemec, \& Lutz 1994; Bersier \& Wood 2001). The first two light curves (top and middle right panels) have asymmetric shape similar to $a b$-type RR Lyrae stars, however they have lower amplitudes by about a factor of two, and the mean magnitudes, calculated from the average of the light curves, are about 0.4 to 1 mag brighter. The curve on the 
last right panel, has a sinusoidal shape similar to $c$-type pulsators, yet its mean magnitude, about 1.4 mag brighter than the RR Lyrae stars, clearly qualifies this star as a Cepheid.

\subsection{Variable stars in the color-magnitude diagram}

The location of the faint variable star sample in the color-magnitude diagram of NGC 6822 is shown in Fig. 2. Only pulsating variable stars with complete light curves and good photometry have been plotted, using their intensity-averaged magnitudes and colors. Different symbols have been used for RR Lyrae stars and more luminous short-period variable stars. Since no HB could be identified even from HST data (Wyder 2001), our detection of RR Lyrae stars provides the first indisputable evidence for the presence of an old population in NGC 6822. In fact, while the HB itself is completely hidden by the overwhelming number of young and intermediate-age stars that populate the same region of the diagram, the position of the RR Lyrae variable stars agrees well with the HB level and the instability strip in the Galactic cluster M 3 (Corwin \& Carney 2001), shifted to the distance and reddening of NGC 6822. We adopted the foreground reddening $E_{B-V}=0.25$ from the maps of Schlegel, Finkbeiner, \& Davis (1998), with no account for internal reddening. The scatter of the data on the red side of the instability strip is probably due to incomplete phase coverage of the light curves.

In addition to RR Lyrae stars, a continuous distribution of short-period variable stars is shown in Fig. 2, filling the region of the diagram above the HB, beginning just about $0.3-$ 0.5 mag brighter than the average luminosity level of the RR Lyrae stars, and with slightly redder mean colors. The observed mean colors and luminosities are not inconsistent with the limits for pulsational instability predicted by theoretical models for the $1.5 M_{\odot}$ models of anomalous Cepheids (Bono et al. 1997).

\section{Analysis}

\subsection{The period-amplitude relation}

Figure 3 shows the period-amplitude relation for the faint variable stars detected in NGC 6822 (namely RR Lyrae and short-period variables fainter than $V=23$ ) for which complete light curves have been obtained.

In several dwarf spheroidal galaxies of the Local Group (Leo II, Leo I, Draco), RR Lyrae stars span a wide range in pulsational properties, suggesting a classification intemediate 
between the Oosterhoff I and II groups (Oosterhoff 1939) of Galactic globular clusters (Siegel \& Majewski 2000; Held et al. 2001; Kinemuchi et al. 2002). In NGC6822, the period and amplitude distributions seem more biased towards an Oosterhoff I type, similarly to RR Lyrae stars in Sculptor (Kaluzny et al. 1995), the LMC (Alcock et al. 1996) and And VI (Pritzl et al. 2002), although a composite distribution is not ruled out by our data. Indeed, the RR Lyrae sample in Fig. 3 is subject to several biases both because RR Lyrae stars are found close to limiting magnitude of our photometry, and because of the short baseline of our observations.

What is the nature of the small-amplitude, short-period variable stars found in NGC 6822 at luminosities only a few tenths of a magnitude brighter than the RR Lyrae stars ? To the best of our knowledge, Cepheids with such low luminosities (LLC), small amplitudes, and short periods (see Fig. 3) have not been found before in any dwarf irregular galaxy, although they are consistent with the predictions of theoretical pulsational models (Fiorentino et al. 2003; F. Caputo et al. 2003, in prep.). Given the extended star-formation history of NGC 6822, these LLC are likely to originate from a population of intermediate-age, metalpoor He-burning stars younger and more massive than the RR Lyrae stars. The brighter variable stars appear more similar to the short-period Cepheids found in other dwarf irregular galaxies (e.g., Dolphin et al. 2003). A full discussion of the characteristics of this population of short-period variable stars, in particular the LLC, is postponed to a forthcoming paper (E. V. Held et al. 2003, in prep.).

\subsection{Metallicity of the old stellar component in NGC 6822}

The mean metallicity of RR Lyrae stars in NGC 6822 can be estimated using the relation between the mean period and metallicity of $a b$-type RR Lyrae stars derived by Sandage (1993) for variable stars in Galactic globular clusters (GGCs), $\log \left\langle P_{a b}\right\rangle=-0.092[\mathrm{Fe} / \mathrm{H}]-$ 0.389. From the average period of the $a b$-type RR Lyrae stars with well-defined light curves, we derived $[\mathrm{Fe} / \mathrm{H}]=-1.92 \pm 0.35$, where the error includes the standard deviation of the measurements, obtained by error propagation from the scatter of the period distribution, and uncertainties due to detection efficiency and possible systematics if RR Lyrae in NGC 6822 are younger than those in GGCs. This value is in remarkable agreement with the low metal abundance measured for an old globular cluster in NGC6822 (Cohen \& Blakeslee 1998; Chandar, Bianchi, \& Ford 2000), and represents the first estimate of the metallicity of the old "field" stellar population in NGC 6822. This value is only slightly more metal-poor than the metal abundance inferred for RGB stars $([\mathrm{Fe} / \mathrm{H}]=-1.5 \pm 0.3$ : Gallart, Aparicio, \& Vilchez 1996a; $-2<[\mathrm{Fe} / \mathrm{H}]<-0.5$ : Tolstoy et al. 2001), which is consistent with a 
plausible chemical enrichment law.

\subsection{The distance to NGC 6822}

The mean magnitude of RR Lyrae variable stars in NGC 6822 provides an independent distance estimate based on Pop. II stars and offers us the possibility to compare the distance scales based on Pop. I and Pop. II indicators. We calculated the mean apparent magnitude of the 6 RR Lyrae variable stars fainter than $V=24.2$ with well-defined light curves and located inside the instability strip. The mean value is $24.63 \pm 0.14$, where the error is the standard deviation of the data.

For the absolute magnitude of RR Lyrae variable stars, we follow here the recent reevalution of Globular Cluster distances by Cacciari \& Clementini (2003), suggesting for the absolute magnitude of RR Lyrae stars $M_{V}(\mathrm{RR})=0.59 \pm 0.03$ at $[\mathrm{Fe} / \mathrm{H}]=-1.5$, corresponding to a distance modulus for the LMC $(m-M)_{0}=18.48 \pm 0.05$. To correct the absolute magnitude to the metallicity of NGC 6822, we adopt the mean metallicity of the old population derived from the mean period of $a b$-type RR Lyrae stars, along with a luminosity-metallicity dependence of 0.22 mag per metallicity dex (as the average of Cacciari \& Clementini 2003 and Clementini et al. 2003). These assumptions yield $M_{V}(\mathrm{RR})=0.50$ for NGC 6822 and a distance modulus $(m-M)_{0}=23.36 \pm 0.17$. The error includes both the systematic error on the photometry zero point and the uncertainty of the mean apparent and absolute magnitude of NGC 6822 RR Lyrae stars, but does not take into account the contribution of possible internal differential reddening.

Table 1 compares this new estimate of the distance to NGC 6822 with distance moduli previously derived using different methods. The new estimate from the RR Lyrae stars agrees well with the distance moduli based on the tip of the red giant branch (Gallart et al. 1996a) and infrared photometry of Cepheids (Mc Alary et al. 1983), while there is a tendency

for the measurements based on the Cepheid period-luminosity relation to yield a somewhat longer distance, a difference that might be ascribed to variable reddening (see, e.g., Venn et al. 2001, and references therein). A similar tendency was noted in other galaxies, most recently by Clementini et al. (2003) and Dolphin et al. (2003).

It is a pleasure to thank M. Marconi for fruitful discussions on stellar pulsation models, and the referee for helpful remarks. We acknowledge support from the National Projects COFIN2001028897 and COFIN2002028935. 


\section{REFERENCES}

Alard, C. 2000, A\&AS, 144, 363

Alcock, C. et al. 1996, AJ, 111, 1146

Bersier, D., \& Wood, P. R. 2002, AJ, 123, 840

Bono, G., Caputo, F., Santolamazza, P., Cassisi, S., \& Piersimoni, A. 1997, AJ, 113, 2209

Cacciari, C., \& Clementini, G. 2003, in Stellar Candles, Lecture Notes in Physics (Berlin:Springer), in press (astro-ph/0301550)

Castelli, F., Gratton, R. G., \& Kurucz, R. L. 1997, A\&A, 318, 841

Chandar, R., Bianchi, L., \& Ford, H. C. 2000, AJ, 120, 3088

Clementini, G. et al. 2000, AJ, 120, 2054

Clementini, G., Gratton, R. G., Bragaglia, A., Carretta, E., Di Fabrizio, L., \& Maio, M. 2003, AJ, 125, 1309

Clement, C. M., \& Rowe, J. 2000, AJ, 120, 2579

Cohen, J. G., \& Blakeslee, J. P. 1998, AJ, 115, 2356

Corwin, T. M., \& Carney, B. W. 2001, AJ, 122, 3183

Dolphin, A. E. et al. 2001, ApJ, 550, 554

Dolphin, A. E. et al. 2002, AJ, 123, 3154

Dolphin, A. E. et al. 2003, AJ, 125, 1261

Fiorentino, G., Caputo, F., \& Marconi, M. 2003, in Stars in Galaxies, eds. M. Bellazzini, A. Buzzoni, S. Cassisi, MSAIt, in press

Gallart, C., Aparicio, A., \& Vilchez, J. M. 1996, AJ, 112, 1928

Gallart, C., Aparicio, A., Bertelli, G., \& Chiosi, C. 1996, AJ, 112, 1950

Held, E. V., Saviane, I., Momany, Y., \& Carraro, G. 2000, ApJ, 530, L85

Held, E. V., Clementini, G., Rizzi, L., Momany, Y., Saviane, I., \& Di Fabrizio, L. 2001, ApJ, 562, L39 
Kaluzny, J., Kubiak, M., Szymanski, M., Udalski, A., Krzeminski, W., \& Mateo, M. 1995, A\&AS, 112, 407

Kinemuchi, K., Smith, H. A., Lacluyzé, A. P., Clark, C. L., Harris, H. C., Silbermann, N., \& Snyder, L. A. 2002, ASP Conf. Ser. 259: IAU Colloq. 185: Radial and Nonradial Pulsationsn as Probes of Stellar Physics (San Francisco:ASP), 130

Landolt, A. U. 1992, AJ, 104, 340

Lee, M. G., Freedman, W. L., \& Madore, B. F. 1993, ApJ, 417, 553

Marconi, G., Tosi, M., Greggio, L., \& Focardi, P. 1995, AJ, 109, 173

McAlary, C. W., Madore, B. F., McGonegal, R., McLaren, R. A., \& Welch, D. L. 1983, ApJ, 273,539

Nemec, J. M., Nemec, A. F. L., \& Lutz T. E. 1994, AJ, 108, 222

Oosterhoff, P. Th. 1939, Observatory, 62, 104

Pritzl, B. J., Armandroff, T. E., Jacoby, G. H., \& Da Costa, G. S. 2002, AJ, 124, 1464

Rejkuba, M., Minniti, D., Gregg, M. D., Zijlstra, A. A., Alonso, M. V., \& Goudfrooij, P. 2000, AJ, 120, 801

Saha, A., Freedman, W. L., Hoessel, J. G., \& Mossman, A. E. 1992a, AJ, 104, 1072

Saha, A., \& Hoessel, J. G. 1990, AJ, 94, 1556

Saha, A., \& Hoessel, J. G. 1990, AJ, 99, 97

Saha, A., Hoessel, J. G., \& Mossman, A. E. 1990, AJ, 100, 108

Saha, A., Hoessel, J. G., \& Krist, J. 1992b, AJ, 103, 84

Sandage, A. 1993, AJ, 106, 687

Schlegel, D. J., Finkbeiner, D. P., \& Davis, M. 1998, ApJ, 500, 525

Schulte-Ladbeck, R. E., Hopp, U., Drozdovsky, I. O., Greggio, L., \& Crone, M. M. 2002, AJ, 124,896

Sharpee, B., Stark, M., Pritzl, B., Smith, H., Silbermann, N., Wilhelm, R., \& Walker, A. 2002, AJ, 123, 3216 
Siegel, M. H., \& Majewski, S. R. 2000, AJ, 120, 284

Stetson, P. B. 1994, PASP, 106, 250

Tolstoy, E., Irwin, M. J., Cole, A. A., Pasquini, L., Gilmozzi, R., \& Gallagher, J. S. 2001, MNRAS, 327, 918

Venn, K. A. et al. 2001, ApJ, 547, 765

Wyder, T. K. 2001, AJ, 122, 2490 
Table 1. Distance determinations to NGC 6822

\begin{tabular}{lllll}
\hline \hline$(m-M)_{0}$ & $E_{B-V}$ & $A_{V}$ & Method & \multicolumn{1}{c}{ Ref. } \\
\hline $23.40 \pm 0.11$ & 0.36 & 1.19 & Ceph & Mc Alary et al. (1983) \\
23.62 & 0.28 & 0.92 & Ceph & Lee et al. (1993) \\
23.46 & 0.28 & 0.92 & TRGB & Lee et al. (1993) \\
$23.49 \pm 0.08$ & 0.24 & 0.80 & Ceph & Gallart et al. (1996a) \\
$23.40 \pm 0.10$ & 0.24 & 0.80 & TRGB & Gallart et al. (1996a) \\
$23.36 \pm 0.17$ & 0.25 & 0.78 & RR Lyrae & this work \\
\hline
\end{tabular}



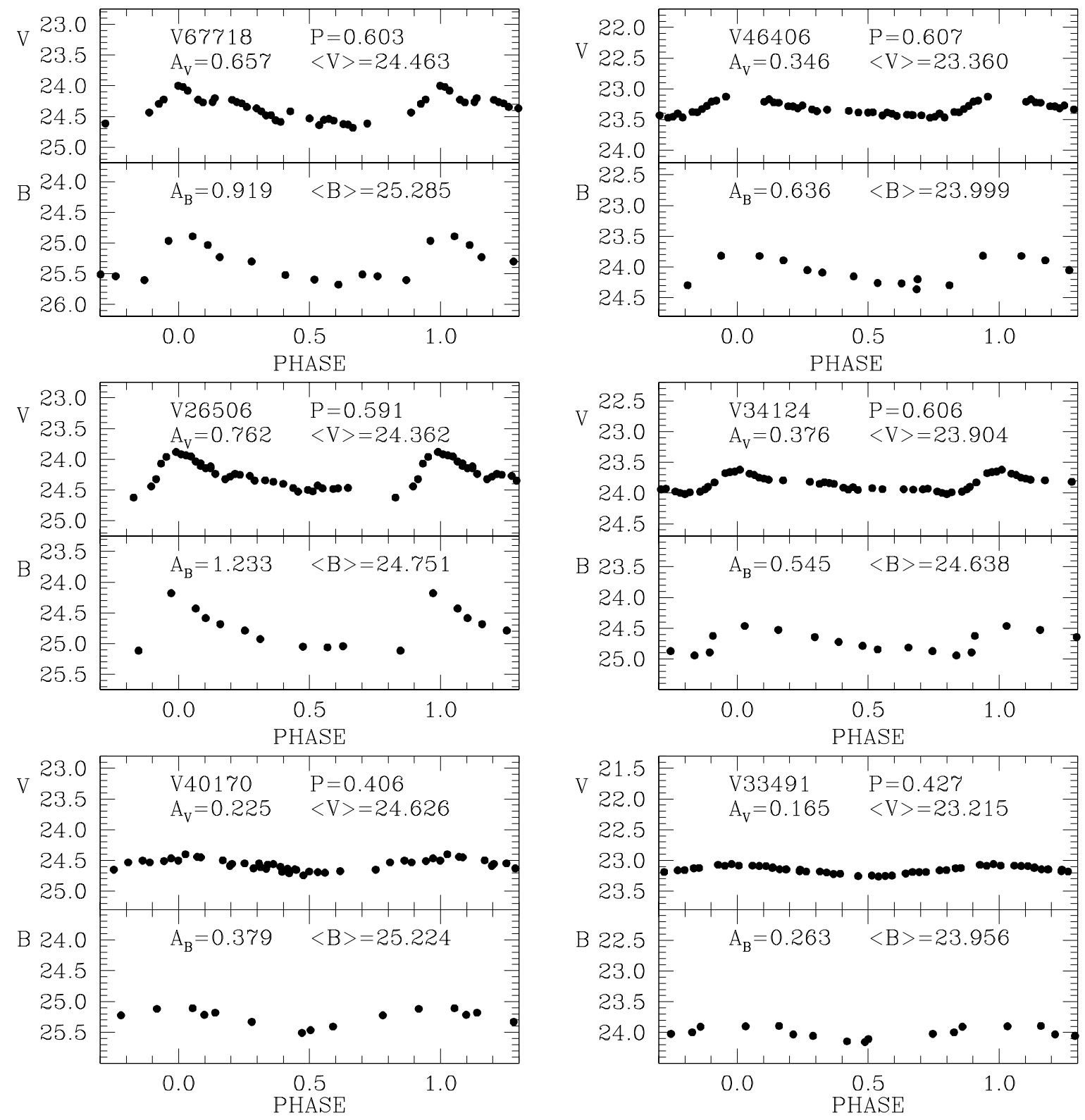

Fig. 1.- $B$ and $V$ light curves of short-period variable stars in NGC 6822. The left panels are RR Lyrae stars, while light curves on the right panels refer to brighter stars. A constant magnitude interval is used in all panels to facilitate the comparison. 


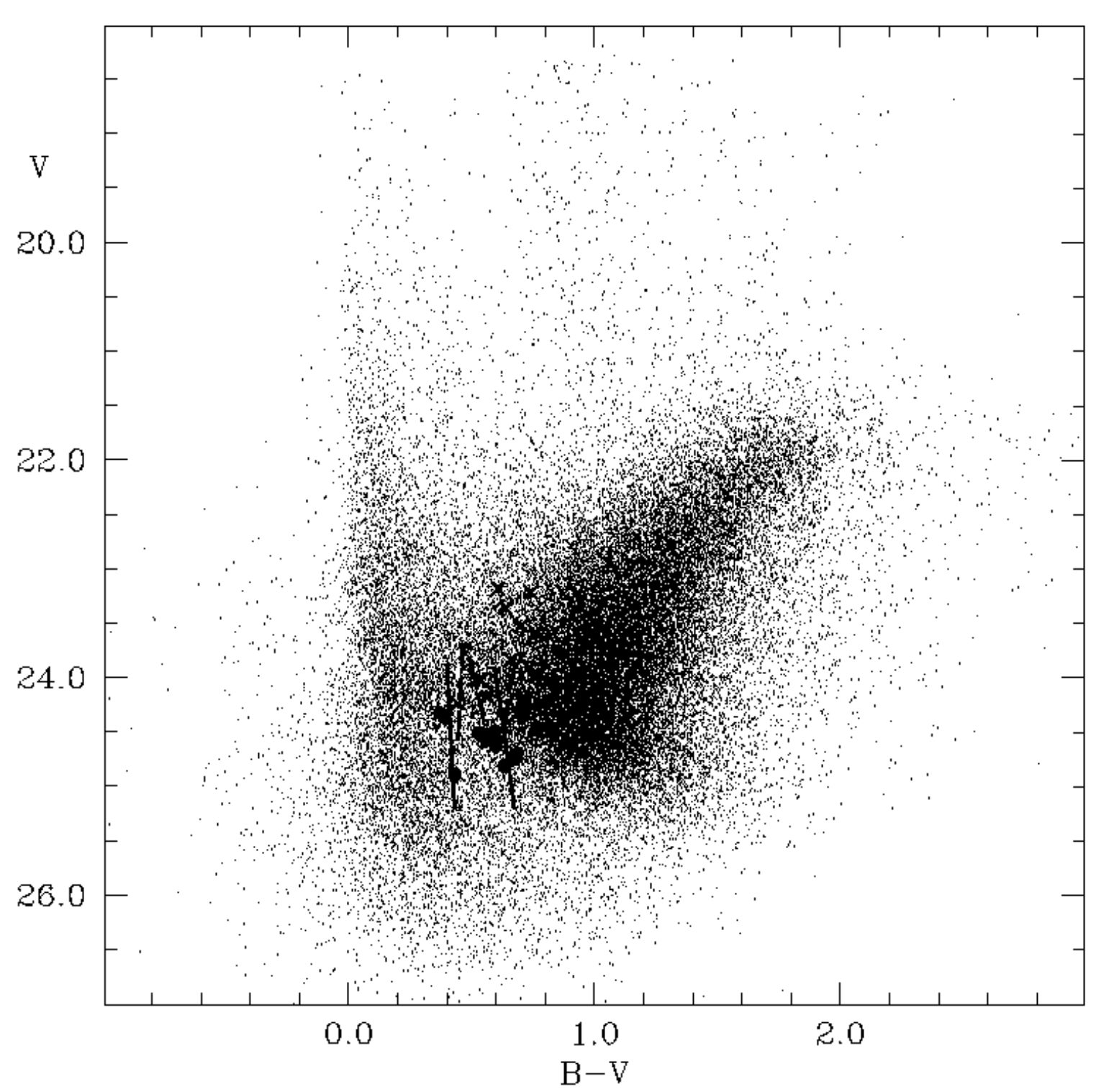

Fig. 2.- The calibrated color-magnitude diagram of NGC6822 showing the location of the newly discovered faint pulsating variable stars. The filled circles are stars classified RR Lyrae, crosses represent small-amplitude, short-period Cepheids. The solid lines show the edges of the instability strip in the globular cluster M 3 (Corwin \& Carney 2001), while the dashed lines are the limits for pulsational instability for $1.5 M_{\odot}$ models (Bono et al. 1997), converted to the observational plane using the model atmospheres of Castelli, Gratton, \& Kurucz (1997). 


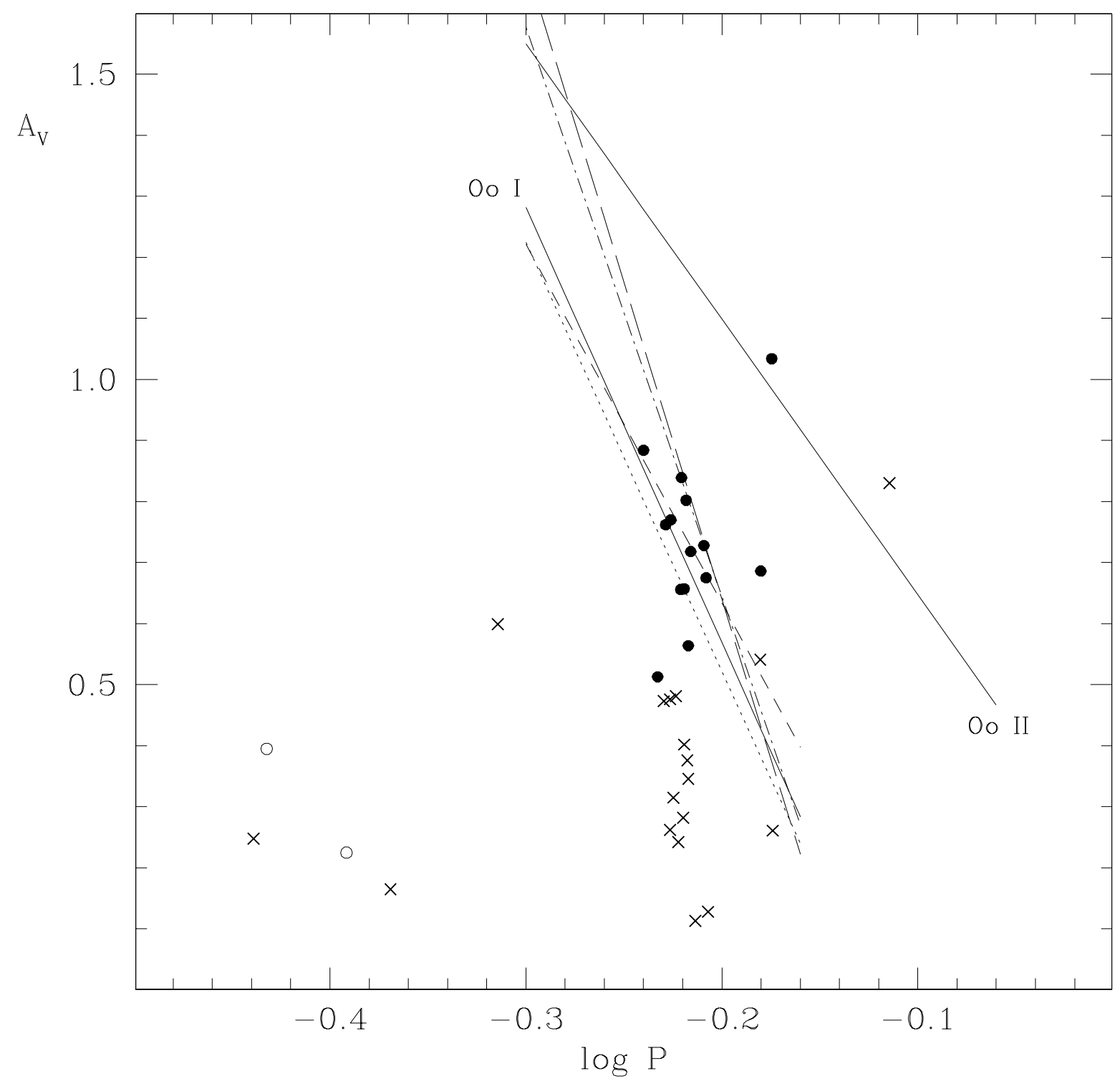

Fig. 3.- Period-amplitude relation for RR Lyrae and other short-period variable stars with complete light curves in NGC6822. Periods are in days. Filled circles and open circles are $a b$-type and c-type RR Lyrae stars in NGC 6822, respectively. The crosses represent the sequence of small-amplitude, short-period Cepheids. The lines show the $A_{V}-\log P$ relations for the ab-type variable stars in Galactic globular clusters (continuous lines, from Clement \& Rowe 2000) and dwarf spheroidal galaxies (And VI: dots; Sculptor: short dash; Leo II: long dash; Draco: dot-short dash; from Pritzl et al. 2002). 